OPEN ACCESS

Edited by:

Nerina Denaro,

Azienda Sanitaria Ospedaliera S.Croce

e Carle Cuneo, Italy

Reviewed by:

Stephen Sonis,

Biomodels LLC, United States

Xu Tian,

University of Rovira i Virgili, Spain

Andrei Barasch,

Harvard Medical Faculty Physicians,

United States

*Correspondence:

Anastasios Maniakas

anastasios.maniakas@umontreal.ca

Specialty section:

This article was submitted to

Head and Neck Cancer,

a section of the journal

Frontiers in Oncology

Received: 27 September 2021 Accepted: 11 November 2021 Published: 02 December 2021

Citation:

Zagury-Orly I, Khaouam N, Noujaim J, Desrosiers MY and Maniakas A (2021) The Effect of Radiation and Chemoradiation Therapy on the Head and Neck Mucosal Microbiome: A Review.

Front. Oncol. 11:784457. doi: 10.3389/fonc.2021.784457

\section{The Effect of Radiation and Chemoradiation Therapy on the Head and Neck Mucosal Microbiome: A Review}

\author{
Ivry Zagury-Orly ${ }^{1}$, Nader Khaouam ${ }^{2}$, Jonathan Noujaim ${ }^{3}$, Martin Y. Desrosiers ${ }^{4,5}$ \\ and Anastasios Maniakas ${ }^{6,7,8^{*}}$
}

${ }^{1}$ Faculty of Medicine, Université de Montréal, Montreal, QC, Canada, ${ }^{2}$ Department of Radiation Oncology, Hôpital Maisonneuve-Rosemont, Montreal, QC, Canada, ${ }^{3}$ Department of Oncology, Hôpital Maisonneuve-Rosemont, Montreal, QC, Canada, ${ }^{4}$ Centre de Recherche du Centre Hospitalier de l'Université de Montréal, Montreal, QC, Canada, ${ }^{5}$ Division of Otolaryngology-Head and Neck Surgery, Centre Hospitalier de l'Université de Montréal, Montreal, QC, Canada, ${ }^{6}$ Division of Otolaryngology-Head and Neck Surgery, Hôpital Maisonneuve-Rosemont, Montreal, QC, Canada, ${ }^{7}$ Department of Experimental Surgery, McGill University, Montreal, QC, Canada, ${ }^{8}$ Department of Head and Neck Surgery, The University of Texas MD Anderson Cancer Center, Houston, TX, United States

Radiation (RT) and chemoradiation therapy (CRT) play an essential role in head and neck cancer treatment. However, both cause numerous side effects in the oral cavity, paranasal sinuses, and pharynx, having deleterious consequences on patients' quality of life. Concomitant with significant advances in radiation oncology, much attention has turned to understanding the role of the microbiome in the pathogenesis of treatment-induced tissue toxicity, to ultimately explore microbiome manipulation as a therapeutic intervention. This review sought to discuss current publications investigating the impact of RT and CRT-induced changes on the head and neck microbiome, using culture-independent molecular methods, and propose opportunities for future directions. Based on 13 studies derived from a MEDLINE, EMBASE, and Web of Science search on November 7, 2021, use of molecular methods has uncovered various phyla and genera in the head and neck microbiome, particularly the oral microbiome, not previously known using culture-based methods. However, limited research has investigated the impact of RT/CRT on subsites other than the oral cavity and none of the studies aimed to examine the relationship between the head and neck microbiome and treatment effectiveness. Findings from this review provide helpful insights on our current understanding of treatment-induced oral mucositis, dental plaque, and caries formation and highlight the need for future research to examine the effect of RT/CRT on the sinonasal and oropharyngeal microbiome. In addition, future research should use larger cohorts, examine the impact of the microbiome on treatment response, and study the effect of manipulating the microbiome to overcome therapy resistance.

Keywords: head and neck cancer, microbiome, radiation therapy, chemoradiation therapy, side effects 


\section{INTRODUCTION}

Head and neck cancers (HNC) are responsible for nearly 330,000 annual deaths worldwide (1). Most HNCs originate from the mucosal epithelium of the upper aerodigestive tract (2). Radiation therapy (RT) is an essential component of HNC treatment. RT alone or combined with chemotherapy [i.e., chemoradiation therapy (CRT)] can be offered as primary treatment, as an adjuvant following surgery, or as palliation for unresectable HNCs (3). The desired tumoricidal effect of RT inadvertently injures normal cells adjacent to the area targeted (4). This results in several side effects, including oral mucositis $(\mathrm{OM})$, xerostomia, dysphagia, odynophagia, and RT-induced chronic rhinosinusitis (CRS), having a significant impact on the quality of life of patients with $\operatorname{HNC}(3,5,6)$.

In efforts of reducing radiation toxicity, significant advances have occurred in the field of RT (4): (i) emergence of new radiation techniques such as intensity-modulated RT; (ii) improvement in optimizing dose schedules, planning, and simulation using functional imaging; and (iii) combining radiation and systemic agents. Other research focuses on improving our molecular understanding of RT-induced tissue injury (4), in part through study of the human microbiome (7).

In this review, we discuss the current research investigating the impact of RT for HNC on the head and neck mucosal microbiome using culture-independent molecular methods and present opportunities for future directions.

\section{MICROBIOME RESEARCH: AN OVERVIEW}

\section{The Basics}

The microbiota refers to the collection of microorganisms in a given environment, whereas the microbiome consists of the microbiota and their genes (8). Metataxonomics is the process by which the microbiota is given its classification. It provides information on the distribution and diversity of microorganisms. This process includes DNA extraction from stool sampling or buccal and nasopharyngeal swabs and subsequent molecular analysis of amplified and sequenced genes, most commonly $16 \mathrm{~S}$ ribosomal RNA (rRNA) genes (for bacteria) and $18 \mathrm{~S}$ rRNA genes (for eukaryotes) $(9,10)$. Viruses, although part of the microbiota, are instead detected by next-generation sequencing (NGS) technologies $(9,10)$, capable of detecting microorganisms at the strain level and analyzing genetic material (metagenomics) that reflect microbiome function (8, 9). Other molecular methods, combined with metagenomics, are increasingly recognized for providing a more integrated analysis (11).

The healthy oral cavity primarily consists of bacterial generaboth gram-positive (e.g., Peptostreptococcus, Streptococcus, Stomatococcus, Actinomyces, Bifidobacterium, Corynebacterium, Lactobacillus, Propionibacterium) and gram-negative (e.g., Moraxella, Neisseria, Veillonella, Campylobacter, Capnocytophaga, Eikenella, Fusobacterium, Hemophilus, Leptotrichia, Prevotella, Treponema)-but also inhabits protozoa (e.g., Entamoeba gingivalis and Trichomonas tenax), fungi (most commonly
Candida species), and viruses (12). A healthy sinonasal microbiome also houses its own set of characteristic genera, namely, Staphylococcus, Propionibacterium, Corynebacterium, and Moraxella (13).

\section{Radiation Therapy and Microbiome Disruption}

The human microbiome contains both beneficial and harmful microorganisms. The adequate balance and interaction between both types enable symbiosis-where both the human and microbiota may benefit-predicting a healthy state, whereas perturbed homeostasis characterizes dysbiosis-a microbial community associated with a diseased state (14). Dysbiosis is believed to be the result of multiple influencing factors-intrinsic (e.g., age, sex, psychological stress, and genetics) and extrinsic (e.g., smoking and alcohol consumption, antibiotics, surgery, RT, and CRT) that perturb the host-microbe-environment interactions (11).

Much of what we currently understand on the effects of RT on the human microbiome derives from gut research (15). A bidirectional effect has been described where RT disrupts the microbiome and through its involvement in mediating immune response, the disrupted microbiome subsequently interferes with RT effectiveness (11). Radiation-induced microbiome disruption is thought to result from two principal mechanisms involving inflammation $(11,16)$. First, radiation directly leads to tissue oxidation and inflammation, which alters the local microenvironment and promotes dysbiosis. Microbiome dysbiosis is thought to disrupt the immune system, leading to an upregulation of pro-inflammatory (e.g., Th17) and downregulation of anti-inflammatory molecules (e.g., regulatory $\mathrm{T}$ cells). Second, radiation causes toxic damage to the epithelium, resulting from cellular DNA and RNA damage, that leads to cell death by apoptosis, followed by ulceration, and exposure of tissue not supposed to be in contact with bacteria, characterized by bacterial translocation and colonization, further increasing the inflammatory response.

Finally, bacteriophages, which are viruses that can kill bacteria, are abundant on epithelial surfaces and are thought to play an important role in human tissue homeostasis and maintenance of health (17). However, to date, these have primarily been discussed in the context of tailored antimicrobial therapy (18), and to our knowledge, metagenomic research assessing the impact of RT on bacteriophages is limited. Nonetheless, we hypothesize that if RT causes a loss of these protective phages, commensal strains may begin showing pathogenic features, or pathogenic strains may become dominant. Such pathogenic species may then readily proliferate in the context of an ulcerated and inflammatory irradiated tissue $(9,18)$.

\section{Clinical Relevance}

The study of microbiome dysbiosis has led to a better understanding of local and systemic disease occurrence. Although the oral microbiome only represents a fraction of the human microbiome, largely represented by the gut (19), oral dysbiosis is linked to periodontitis, dental caries, and HNC, but also to endocarditis, atherosclerosis, Alzheimer's disease, and 
diabetes (20). Similarly, gut dysbiosis has been associated with gastrointestinal disease occurrence (e.g., inflammatory bowel disease, irritable bowel syndrome, and clostridium difficile infection), as well as neurodegenerative disease, diabetes, rheumatoid arthritis, and obesity $(14,15)$. The gut microbiome is also linked to HNC; symbiosis leads to favorable outcomes whereas dysbiosis has been associated with the development of psychoneurological symptoms related to cancer treatment (21). Moreover, specific microorganisms may be associated with refractory disease. For example, the presence of Staphylococcus aureus in the sinonasal microbiome appears to predict failure of endoscopic sinus surgery in patients with CRS at high risk of recurrence (22). Identification of such microorganisms, then allows for clinical trials that measure the effectiveness of specific antibiotic regimens in reducing the abundance of harmful pathogens in patients with refractory disease such as CRS (23).

As we will explore in this review, therapeutic modalities that do not target the microbiome intentionally, such as RT or CRT, cause changes in the microbiota that may perturb an otherwise symbiotic environment and help explain decreased treatment efficacy and undesirable side effects. Understanding how and why the microbiome is modified in response to RT has led to the emergence of probiotics and fecal microbial transplantation used to recreate an appropriate balance of pro-and anti-inflammatory cells with aims of enhancing response to RT and minimizing toxicity $(11,24)$.

\section{RADIATION THERAPY AND CHANGES IN THE HEAD AND NECK MICROBIOME}

A search was conducted on the MEDLINE (Table 1), EMBASE, and Web of Science databases on November 7, 2021, combining the following concepts: (i) head and neck malignancy, (ii) radiation therapy, and (iii) microbiome, using various keywords. A total of 378 studies were screened, 13 of which were included in our qualitative synthesis (Table 2). Studies were excluded if any of the concepts included in the search were not central to the article or if they used culture-based methods.
Until the last decade, microbial research in irradiated HNC patients was entirely culture-based $(3,37)$. Cultivation-based analyses greatly limit the identification of microbial species, thus providing significantly less information on biodiversity and polymicrobial environments and no data on the function of the microbiome (38). Additionally, cultures are time-consuming and thus non-desirable when speedy identification is warranted (39).

Nonetheless, much of what we know about the effects of RT on head and neck microbiota, especially in the oral cavity, is due to culture-based methods. There is convincing evidence to suggest that RT modifies the composition of oral microbiota, mainly with an increase in the number of gram-negative bacteria [e.g., Klebsiella sp (40, 41), Pseudomonas aeruginosa (41)], Candida albicans (40-44), and some gram-positive bacteria, most importantly Lactobacillus sp $(42,43,45)$. While prior research supports the association between microorganisms identified in RT-treated oral cavities of HNC patients and RT-induced toxicity $(5,45-48)$, the behaviors and functions of the head and neck microbiome (HNM) have yet to be fully understood as a potential explanatory mechanism (16). In a randomized clinical trial, Stokman et al. sought to assess the impact of selective microbiota elimination using topical broad-spectrum antibiotic treatment (polymyxine E, tobramycin, and amphotericin B) compared to placebo on the development of radiation-induced OM (47). The authors concluded that selective elimination is not effective in preventing the development of OM. Such findings have formed the basis for the research presented from here onwards, using culture-independent molecular analyses.

Multiple RT and CRT-induced changes in HNM have been discovered in the past decade using molecular analyses. An increased number and diversity of fungi, in addition to Candida, have been detected (e.g., Peniophora, Stereum, Cladosporium) (3). The total number of bacteria tends to decrease immediately after treatment and gradually return, while the relative abundance of certain species (e.g., Streptococcus mutans) and genera (e.g., Bifidobacterium, Lactobacillus) tend to increase and reflect gut-associated obligate anaerobes $(3,27,28,33,35)$. Regarding archea, there

TABLE 1 | MEDLINE Search Strategy.

\begin{tabular}{|c|c|c|c|}
\hline Concepts & \# & Searches & $\begin{array}{c}\text { Results } \\
\text { (November } \\
7,2021 \text { ) }\end{array}$ \\
\hline A & 2 & $\begin{array}{l}\text { ("ear nose and throat" OR otorhinolaryngolog* OR otolaryngolog* OR otolog* OR laryngolo* OR rhinolog* OR ent OR otl OR oral OR nasal } \\
\text { OR sinus OR sinonasal OR ear).ab,ti }\end{array}$ & 991257 \\
\hline & 4 & or/1-3 & 1247980 \\
\hline B & 5 & $\begin{array}{l}\text { exp radiotherapy/OR exp radiofrequency therapy/su OR exp chemoradiotherapy/su OR exp radioimmunotherapy/su OR [radi adj1 } \\
\text { (therap*)].ab,ti OR [(radiotherap* OR irradiat* OR postradiation)].ab,ti }\end{array}$ & 552683 \\
\hline \multirow[t]{2}{*}{ C } & 6 & exp microbiota/OR (microbiom).ab,ti & 69893 \\
\hline & 7 & 4 and 5 and 6 & 58 \\
\hline
\end{tabular}

The asterisk (*) represents any group of characters, including no character. 
TABLE 2 | Impact of Radiation or Chemoradiation Therapy on the Head and Neck Microbiome.

\begin{tabular}{|c|c|c|c|c|c|}
\hline Source & Aim & $\begin{array}{l}\text { Treatment Modality } \\
\text { (sample Size) }\end{array}$ & $\begin{array}{c}\text { Bacterial } \\
\text { identification }\end{array}$ & Observations & Main limitations \\
\hline
\end{tabular}

\section{Oral cavity}

Saliva

Xu et al

(25)

Characterize the oral microbiota of CRT $(n=3)$ for NPC;

Zhang et al Investigate the relationship

(26) between salivary function, oral

microbiota, and radiation caries

Kumpitsch Characterize change in bacterial,

et al (3) fungal, and archaeal components of the salivary microbiome

\section{Buccal mucosa}

Mougeot Determine changes in oral

et al (27) microbiome across RT treatment and characterize association with dental caries

Reyes- Determine changes in oral

Gibby et al microbiota on OM onset,

(28) incidence, and severity

\section{Saliva and buccal mucosa}

Vesty et al Determine changes in oral

(29) microbiota on OM incidence and severity

\section{Supragingival plaque}

$\mathrm{Hu}$ et al Study variations in oral microbiota

(30) of supragingival plaque during RT

Gao et al Study variations in oral microbiota $\mathrm{RT}(\mathrm{N}=3)$ for HNC

(31) of supragingival plaque before and after RT

\section{Pharynx: Oropharyngeal swab}

Hou et al Determine changes in oral

(32) microbiota on OM incidence and severity

Oliva et al Characterize oral and gut

(33) microbiome pre- and post- CRT in HPV+ patients with OPSCC
Historical controls $(n=3)$
RT without caries $(n=12)$; $R T$, with caries $(n=9)$ for NPC

CRT $(n=31)$ for HNSCC; Control $(n=11)$

RT (N = 31) for HNSCC

CT only ( $n=2)$; RT only $(n=7)$; CRT $(n=57)$ for HNSCC

$\mathrm{RT}(\mathrm{N}=8)$ for $\mathrm{HNC}$ patients HPV+

RT alone ( $n=3)$; CRT $(n=16)$ for NPC
RT $(\mathrm{N}=19)$ for HNSC

16S rRNA gene

amplicon

pyrosequencing

(Roche 454 GS-FLX)

16S rRNA gene

amplicon sequencing

(Applied Biosystems)

16S rRNA gene and

ITS amplicon

sequencing (Illumina

MiSeq)

16S rRNA gene

amplicon sequencing (modified Illumina

MiSeq)

16S rRNA gene amplicon sequencing (Illumina MiSeq)

16S rRNA gene and ITS amplicon sequencing (lllumina MiSeq)

16S rRNA gene amplicon pyrosequencing (Roche 454 GS-FLX) 16S rRNA gene amplicon pyrosequencing (Roche 454 GS-FLX)

16S rRNA gene amplicon sequencing (Illumina HiSeq2000)

16S RNA and shotgun metagenomic sequencing (Illumina Nextera Flex)
- $\uparrow$ Neisseria, Leptotrichia and Pseudomonas, and $\downarrow$ Streptococcus in NPC vs. controls

- Changes in salivary function and oral microbiota post-RT do not explain the absence of radiation caries in radiation caries-free individuals

- $\uparrow$ fungi (e.g., Candida)

- $\downarrow$ bacteria (relative $\uparrow$ Bifidobacterium) - No change in archeal composition

- Significant changes in RT-treated oral microbiome (e.g., $\uparrow$ Streptococcus mutans) - $\uparrow$ Tooth decay associated with $\downarrow$ Abiotrophia defective

- Caries not associated with a difference in salivary flow

- $86 \%$ of patients developed OM, median onset 21 days

- $\uparrow$ Genera of normal microbiota (varying throughout the course of treatment) associated with onset and severity of OM

- $\uparrow$ Candida, not associated with OM incidence and severity

- GNB: some positively correlated with OM incidence; others, with OM severity - Little variation throughout the course of treatment

- Negative correlation between operational taxonomic units and radiation dose

- $\uparrow$ RT dose results in $\downarrow$ diversity and richness of the oral microbiome.

- Gradual return to pre-RT microbiome post-RT completion

- Genera in OM strongly correlated with genera in periodontal disease

- Prevotella, Fusobacterium, Treponema, and Porphyromonas varied in abundance throughout RT: peak associated with the onset of severe OM

- $\downarrow$ number of species and $\uparrow$ relative abundance of gut-associated obligate anaerobes in oropharyngeal swabs
- Limited sample size with inter-

subject variability - Inadequately matched controls - Study-design (cross-sectional)

- Limited sample size

- Heterogeneity of subjects (treatment and demographics)

- Limited sample

size

- Inability to

measure strain-level differences

- Limited sample size

- Dietary changes during therapy not controlled for

- Limited sample size - Study design (no control group)

- Possible sampling error (site-specific oral microbiome)

- Limited sample size

- Data analysis method (inability to detect fungi)

- Limited sample

size

- Data analysis method (poor understanding of temporal dynamics) - Limited sample size

- Possible unaccounted confounders (e.g. dietary habits) 
TABLE 2 | Continued

\begin{tabular}{|c|c|c|c|c|c|}
\hline Source & Aim & $\begin{array}{l}\text { Treatment Modality } \\
\text { (sample Size) }\end{array}$ & $\begin{array}{c}\text { Bacterial } \\
\text { identification }\end{array}$ & Observations & Main limitations \\
\hline $\begin{array}{l}\text { Zhu et al } \\
\text { (34) }\end{array}$ & $\begin{array}{l}\text { Determine changes in oral } \\
\text { microbiota on } \mathrm{OM} \text { incidence and } \\
\text { severity }\end{array}$ & $\begin{array}{l}\text { RT only }(n=17) \text {; Induction } \\
\text { CRT + RT ( } n=2) ; \text { CRT + } \\
\text { CT }(n=23) \text { for NPC; } \\
\text { Control }(n=49)\end{array}$ & $\begin{array}{l}\text { 16S rRNA gene } \\
\text { amplicon sequencing } \\
\text { (Illumina HiSeq2000) }\end{array}$ & $\begin{array}{l}\text { - } \downarrow \text { bacterial diversity and } \uparrow \text { Actinobacillus, } \\
\text { Mannheimia and Streptobacillus, and } \\
\text { unclassified Pasteurellales and } \\
\text { pasteurellaceae } \\
\text { associated with increased OM severity }\end{array}$ & $\begin{array}{l}\text { - Limited sample } \\
\text { size }\end{array}$ \\
\hline $\begin{array}{l}\text { Lim et al } \\
(35)\end{array}$ & $\begin{array}{l}\text { Determine changes in oral } \\
\text { microbiome and metabolomic } \\
\text { profiles up to } 24 \text { months post- } \\
\text { CRT }\end{array}$ & $\begin{array}{l}\text { RT or CRT for OCC } \\
(n=9) \text { and OPC }(n=20)\end{array}$ & $\begin{array}{l}16 S \text { rRNA gene } \\
\text { amplicon sequencing } \\
\text { (lllumina MiSeq) }\end{array}$ & $\begin{array}{l}\text { - Most genera } \downarrow \text { immediately post-treatment } \\
\text { and most gradually return to pre-radiation } \\
\text { levels } \\
\text { - } \uparrow \text { relative abundance of Lactobacillus and } \\
\text { Streptococcus immediately post-CRT } \\
\text { - downregulation of nitric oxide metabolites } \\
\text { post-CRT in saliva samples }\end{array}$ & $\begin{array}{l}\text { - Limited sample } \\
\text { size }\end{array}$ \\
\hline \multicolumn{6}{|c|}{ Nose and Paranasal Sinuses: Sinonasal swab } \\
\hline $\begin{array}{l}\text { Stoddard } \\
\text { et al (36) }\end{array}$ & $\begin{array}{l}\text { Describe and compare the } \\
\text { sinonasal microbiota of } \\
\text { rhinosinusitis after RT using } \\
\text { culture and molecular techniques }\end{array}$ & $\begin{array}{l}\mathrm{RT}(\mathrm{N}=22) \text { for } \mathrm{SN}, \mathrm{NP} \\
\text { and } \mathrm{SB} \text { cancer ( } 9 \text { types) }\end{array}$ & $\begin{array}{l}16 S \text { rRNA gene } \\
\text { pyrosequencing } \\
\text { (Micro GenX) vs. } \\
\text { culture }\end{array}$ & $\begin{array}{l}\text { - Molecular analyses are superior to culture } \\
\text { ( } \uparrow \text { number and diversity of microorganisms) } \\
\text { - Post-RT sinusitis microbiota was similar to } \\
\text { that of chronic sinusitis in healthy adults }\end{array}$ & $\begin{array}{l}\text { - Limited sample } \\
\text { size } \\
\text { - Study design } \\
\text { (retrospective) }\end{array}$ \\
\hline
\end{tabular}

CRT, chemoradiotherapy; CT, chemotherapy; GNB, gram-negative bacteria; HN, head and neck; HNC, head and neck cancer; HNSCC, head and neck squamous cell carcinoma; NP, nasopharyngeal; NPC, nasopharyngeal carcinoma; ITS, GNB, gram-negative bacteria; OCC, oral cavity cancer; OM, oral mucositis; OPC, oropharyngeal cancer; OPSCC, oropharyngeal squamous cell carcinoma; rRNA, ribosomal RNA; RT, radiotherapy; SB, skull base; SN, sinonasal. Symbols: $\uparrow$, increase; $\downarrow$, decrease.

seems to be little to no change due to RT (3). Furthermore, recent studies have investigated the impact of varying radiation doses, noting an inverse relationship between dose and microbiome diversity $(31,37)$. Additionally, much of recent research has characterized the HNM of patients with evidence of RT-induced tissue toxicity (e.g., OM) $(28,29,32,34)$. Other studies within the oral cavity have investigated the variation of RT-induced changes in the microbiome of supragingival plaque in HNC patients (30, 31 ), and possible associations with the incidence of dental caries $(26,27)$.

\section{Oral Mucositis}

$\mathrm{OM}$ is one of the most incapacitating side effects of RT, CT, or CRT for HNC and thus one of the most extensively studied (5, 49-51). The World Health Organization's OM Toxicity Scale ranges from grade 1 to 4: (1) soreness with or without erythema, (2) ulcers with erythema without dysphagia to solid foods, (3) ulcers with extensive erythema and dysphagia to solid foods, and (4) mucositis with complete dysphagia (5). Grades 3 and 4 are considered severe. Nearly $90 \%$ of HNC patients receiving RT develop OM, with more than two-thirds of cases classified as severe $(5,49)$.

In the traditional model for understanding the pathophysiology of therapy-induced OM, radiation causes tissue oxidation, DNA damage, and cell death, followed by a pro-inflammatory response causing secondary tissue damage; this leads to ulcer formation with translocation of bacteria leading to more inflammation, and subsequent healing (52, 53). However, this model should no longer form the sole basis for understanding OM pathophysiology due to the passive role it attributes to the oral microbiome, which is known to modulate the immune system $(54,55)$. Radiation-induced dysbiosis is thought to disrupt the immune response, which may affect the development and persistence of ulcerations (28).
In aims of bettering our understanding of $\mathrm{OM}$ pathophysiology, incidence, and severity, studies have described changes in oral microbiome relative abundance and diversity post-exposure to RT/CRT. Zhu et al. (34) studied the oral microbiome of 41 patients receiving $\mathrm{RT}$ alone or in various combinations with CT for nasopharyngeal carcinoma (NPC). They noted that a decreased bacterial diversity and an increased abundance of Actinobacillus, Mannheimia, Streptobacillus, and unclassified Pasteurellales and pasteurellaceae were associated with increased OM severity. Interestingly, Vesty et al. (29) found that an increased abundance of Candida was not associated with OM incidence nor severity, but that specific gram-negative bacteria (e.g., Fusobacterium, Haemophilus) were associated with $\mathrm{OM}$ susceptibility, and that others (e.g., Porphyromonas and Tannerella) were associated with greater OM severity. In another study (32), the peak in abundance of similar microorganisms (e.g., Prevotella, Fusobacterium, Treponema, and Porphyromonas), found in necrotizing ulcerative gingivostomatitis (53), were associated with the onset of severe OM. The authors in the latter study reinforced the importance of culture-independent techniques by identifying uncultivable pathogens (e.g., Filifactor, Selenomonas, Parvimonas, Peptostreptococcus) found in periodontal disease (32). Finally, a recently published study (28) noted an onset of $\mathrm{OM}$ at 21 days post-CRT and identified different genera at varying stages of OM progression: Prevotella, Fusobacterium, and Streptococcus immediately before OM appearance, and Megasphaera and Cardiobacterium immediately before the development of severe OM.

\section{Dental Plaque and Caries Formation}

Dental plaque is composed of a diverse microbiota. In symbiosis (i.e. microbial homeostasis), neighboring oral tissue (e.g., teeth) remain healthy (56). Factors such as 
inadequate salivary flow and diet intake can modify the microbial habitat, which in combination with numerous other genetic, immunological, environmental, and behavioral factors leads to dysbiosis, which favors the growth of dental plaque and caries formation $(57,58)$. Dental plaque is primarily composed of Firmicutes and Proteobacteria phyla and gram-negative, anaerobic species such as Veillonella parvula, Streptococcus oralis, and Streptococcus mutans that favor tartar formation, dental caries, and periodontal disease $(56,58)$.

Several studies have sought to investigate the changes of the dental plaque microbiome in different pathological states. $\mathrm{Hu}$ and colleagues (30) studied the oral microbiome of supragingival plaque during RT for HNC and found a negative correlation between operational taxonomic units and radiation dose. This finding was corroborated by a study in 2015 by Gao and colleagues (31), who noted that as RT dose increased, the diversity and richness of the oral microbiome in supragingival plaque decreased during RT but gradually returned to normal post-RT completion.

Thus, RT appears to perturb plaque composition only temporarily. Whether such RT-induced changes lead to dental caries formation has previously been studied. Zhang et al. (26) compared two groups, both had NPC and underwent RT. One group had RT-induced caries $(n=12)$, the other was caries-free $(n=9)$. The oral microbiome was compared to determine its relationship with the presence or absence of carious lesions. These authors found that oral microbiome post-RT could not explain the absence of radiation caries in radiation caries-free individuals. Another study noted that increased tooth decay was associated with decreased Abiotrophia defective, a potentially protective oral gram-positive bacteria (27). Thus, compared to $\mathrm{OM}$, the relative $\mathrm{RT}$-induced microbiome alterations as explanatory mechanisms for dental caries occurrence seem to have less of a role or have yet to be adequately elucidated.

\section{Effect of Head and Neck RT on Non-Oral Cavity Subsites}

Only a single study (36) investigated RT-induced microbial changes outside the oral cavity and oropharynx. In 2019, Stoddard et al. described and compared the sinonasal microbiota of rhinosinusitis post-RT using culture-based and molecular techniques (36). They collected sinonasal DNA samples of 22 patients with one of nine types of sinonasal, nasopharyngeal, and skull-based neoplasms. The authors recognized molecular analyses as superior to culture-based methods due to their capacity in detecting a greater number and diversity of microorganisms; albeit, both methods identified Staphylococcus aureus as the most common organism followed by Pseudomonas aeruginosa. They noted that the post-RT sinusitis microbiota was similar to that of CRS in healthy adults (36).

Although data on RT and the microbiome of non-oral cavity subsites is limited, several studies of the HNM without relation to RT are available; interested readers are referred to recent reviews, discussing the ear (59), nose (60), pharynx, and larynx $(61,62)$.

\section{THE HEAD AND NECK MICROBIOME AND RESPONSE TO TREATMENT}

The microbiome also plays a key role in predicting RT efficacy. Studies from the gut microbiome suggest tumor-induced dysbiosis prior to RT and RT-induced microbiome perturbation as mechanistic explanations for reduced RT effectiveness $(11,16,63,64)$. Although severe RT-induced toxicity (e.g., severe $\mathrm{OM}$ ) can limit treatment completion and success (64), no study aimed at determining RT effectiveness as a function of the HNM was identified.

Understanding the role of the microbiome in cancer treatment has several clinical implications. One such implication is modifying the HNM to prevent the development of severe OM (5). For example, a recent randomized controlled trial (65) of 99 patients with NPC undergoing CRT found that patients who received concomitant probiotics during CRT had a significant decrease in the incidence of severe OM compared to controls who had CRT alone ( $15.5 \%$ vs. $45.7 \%$, respectively). Analysis with $16 \mathrm{~S}$ rRNA gene sequencing before and after CRT indicated that the use of probiotics restored microbial diversity to that of healthy individuals. Other studies observed that a reduction in severe $\mathrm{OM}$ due to $\mathrm{RT}$ is associated with higher rates of treatment completion (64).

\section{FUTURE DIRECTIONS}

The findings discussed in this review have uncovered several opportunities for future study of the HNM in response to RT/ CRT, both from a methodological and therapeutic perspective.

The studies we present have methodological advantages over culture-based techniques: superior sensitivity, specificity, and speed in detecting microorganisms that may be responsible for causing RT-induced toxicity $(39,66)$. By using "sequencing by synthesis" methods that target the 16S rRNA subunit (67), studies in this review allow for identification of microorganisms at the phyla and genera levels, while one study (33) used metagenomic shotgun sequencing to capture species-level changes. Recent advancements in molecular processing have made possible the use of a multi-omic approach-metagenomics, metabolomics, metatranscriptomics, and metaproteomics (68) - to identify strain-level microorganisms and obtain additional information on how the microbiome may be related to clinical changes in the host $(27,69)$.

Additionally, study of the HNM in patients undergoing RT/ CRT has primarily sought to characterize dysbiosis and establish a possible link with patient symptomatology, particularly in severe OM. However, limited research is available on whether RT-induced changes in the HNM are responsible for resistance to treatment and why this may occur. Nevertheless, targeted therapy is actively being investigated for HNCs resistant to treatment. One example is the emerging use of immune checkpoint inhibitors (immunotherapy drugs that prevent immune checkpoints proteins from inhibiting $\mathrm{T}$ cells intended to destroy cancer cells) as first-line treatment or in combination 
with RT/CRT (70). Moreover, bacteriophage therapy, a type of personalized medicine that spares healthy cells-unlike broadspectrum antibiotics (18) - are now being studied in the HNM of non-cancer patients (71). Additionally, although not yet studied in the HNM (72), the use of microbiota transplantation from responsive donors may increase response to immunotherapy, given the established role of the microbiome in modulating immune response (73).

One important challenge in studying the oral microbiome is that the diversity of microorganisms is both individual- and sitespecific. In the oral cavity, specific microorganisms preferentially reside in certain microbial loci or sub-niches (e.g., tongue, cheek, and teeth) (74). For example, the microbiome of subgingival plaque differs from that of the resident dorsal tongue, and the tongue has a different microbial diversity than the buccal mucosa (12). Such specificity also exists within a given oral cavity structure; for example, the microbiome of subgingival plaque varies according to the tooth surface (75). Hence, researchers should carefully define the intraoral site for which data is collected and analyzed.

Our review noted that most studies were focused on the oral microbiome, whereas a single study (36) investigated the impact of RT on the sinonasal microbiome. Approximately one-sixth of patients undergoing RT for NPC will experience RT-induced rhinosinusitis (76), believed to be secondary to tissue injury, inflammation, and edema to the sinonasal cilia and mucussecreting cells (77-79). Thus, despite the frequency of tissue toxicity, the impact of RT/CRT on the sinonasal microbiome remains understudied.

Therefore, future studies should characterize the sinonasal microbiome of patients treated with RT/CRT, and prospectively evaluate patients during treatment, using molecular methods, while considering the use of a multi-omic approach to detect change over time at the strain level. Furthermore, microbiome dysbiosis identified in preliminary studies should be considered with respect to patient symptomatology and response to treatment. Although this review explored dysbiosis in relation to cancer therapy, dysbiosis due to various intrinsic and extrinsic factors is associated with cancer development through

\section{REFERENCES}

1. Siegel RL, Miller KD, Fuchs HE, Jemal A. Cancer Statistics, 2021. CA Cancer J Clin (2021) 71(1):7-33. doi: 10.3322/caac.21654

2. Johnson DE, Burtness B, Leemans CR, Lui VWY, Bauman JE, Grandis JR. Head and Neck Squamous Cell Carcinoma. Nat Rev Dis Primers (2020) 6 (1):92. doi: 10.1038/s41572-020-00224-3

3. Kumpitsch C, Moissl-Eichinger C, Pock J, Thurnher D, Wolf A. Preliminary Insights Into the Impact of Primary Radiochemotherapy on the Salivary Microbiome in Head and Neck Squamous Cell Carcinoma. Sci Rep (2020) 10 (1):16582. doi: 10.1038/s41598-020-73515-0

4. Citrin DE. Recent Developments in Radiotherapy. Longo DL, Ed. N Engl J Med (2017) 377(11):1065-75. doi: 10.1056/NEJMra1608986

5. Maria OM, Eliopoulos N, Muanza T. Radiation-Induced Oral Mucositis. Front Oncol (2017) 7:89 doi: 10.3389/fonc.2017.00089

6. Feng S, Fan Y, Liang Z, Yang G, Liao Z, Guo L. Effect of Intranasal Steroids on Rhinosinusitis After Radiotherapy for Nasopharyngeal Carcinoma: Clinical Study. J Laryngol Otol (2016) 130(3):265-71. doi: 10.1017/S0022215115003448 inflammation and may, in part, be caused by cancer itself ( 72 , 80); identification of possible pathophysiological mechanisms linking this complex relationship should be a core objective of future research, allowing for the eventual proposition and assessment of therapeutic modalities (e.g., probiotics) and ultimately tailored care. Finally, future research should be conducted with adequately powered populations, as discussed by several studies $(3,25,27-29,31,32,34-36)$, who, for the most part, stated the need for larger sample sizes.

\section{CONCLUSIONS}

The study of the microbiome in HNC as an explanatory mechanism and potential therapeutic avenue for overcoming the limitation of locoregional toxicity and response of RT and CRT has gained significant interest in recent years. Analysis of the HNM using molecular methods has uncovered various microorganisms not previously known using culture-based methods, providing helpful insights into RT-induced OM, dental plaque, and caries formation. Our review highlights the need for future research to (i) explore further the effect of RT and CRT using molecular techniques, on not only the oral microbiome but also on the sinonasal and oropharyngeal microbiome, in adequately powered populations; (ii) to identify if dysbiosis was accompanied by patient symptomatology and response to treatment and explore mechanisms by which these occur; and finally (iii) to propose and investigate potential modes of intervention.

\section{AUTHOR CONTRIBUTIONS}

IZ-O, MD, and AM contributed to conceptualization and design. All authors contributed to analysis and interpretation of data. IZ-O and AM drafted the manuscript. All authors critically revised the manuscript for important intellectual content and approved it for publication.

7. Cullen CM, Aneja KK, Beyhan S, Cho CE, Woloszynek S, Convertino M, et al. Emerging Priorities for Microbiome Research. Front Microbiol (2020) 11:136. doi: $10.3389 /$ fmicb.2020.00136

8. Ursell LK, Metcalf JL, Parfrey LW, Knight R. Defining the Human Microbiome. Nutr Rev (2012) 70(Suppl 1):S38-44. doi: 10.1111/j.17534887.2012.00493.x

9. Marchesi JR, Ravel J. The Vocabulary of Microbiome Research: A Proposal. Microbiome (2015) 3(1):31. doi: 10.1186/s40168-015-0094-5

10. Breitwieser FP, Lu J. Salzberg SL. A Review of Methods and Databases for Metagenomic Classification and Assembly. Briefings Bioinf (2019) 20 (4):1125-36. doi: 10.1093/bib/bbx120

11. Liu J, Liu C, Yue J. Radiotherapy and the Gut Microbiome: Facts and Fiction. Radiat Oncol (2021) 16(1):9. doi: 10.1186/s13014-020-01735-9

12. Deo PN, Deshmukh R. Oral Microbiome: Unveiling the Fundamentals. J Oral Maxillofac Pathol (2019) 23(1):122-8. doi: 10.4103/jomfp.JOMFP_ 304_18

13. Rawls M, Ellis AK. The Microbiome of the Nose. Ann Allergy Asthma Immunol (2019) 122(1):17-24. doi: 10.1016/j.anai.2018.05.009 
14. Shreiner AB, Kao JY, Young VB. The Gut Microbiome in Health and in Disease. Curr Opin Gastroenterol (2015) 31(1):69-75. doi: 10.1097/ MOG.0000000000000139

15. Cani PD. Human Gut Microbiome: Hopes, Threats and Promises. Gut (2018) 67(9):1716-25. doi: 10.1136/gutjnl-2018-316723

16. Tonneau M, Elkrief A, Pasquier D, Paz Del Socorro T, Chamaillard M, Bahig $\mathrm{H}$, et al. The Role of the Gut Microbiome on Radiation Therapy Efficacy and Gastrointestinal Complications: A Systematic Review. Radiother Oncol (2021) 156:1-9. doi: 10.1016/j.radonc.2020.10.033

17. Sausset R, Petit MA, Gaboriau-Routhiau V, De Paepe M. New Insights Into Intestinal Phages. Mucosal Immunol (2020) 13(2):205-15. doi: 10.1038/ s41385-019-0250-5

18. Clokie MRJ, Millard AD, Letarov AV, Heaphy S. Phages in Nature. Bacteriophage (2011) 1(1):31-45. doi: 10.4161/bact.1.1.14942

19. Lloyd-Price J, Abu-Ali G, Huttenhower C. The Healthy Human Microbiome. Genome Med (2016) 8(1):51. doi: 10.1186/s13073-016-0307-y

20. Radaic A, Kapila YL. The Oralome and Its Dysbiosis: New Insights Into Oral Microbiome-Host Interactions. Comput Struct Biotechnol J (2021) 19:133560. doi: 10.1016/j.csbj.2021.02.010

21. Bai J, Bruner DW, Fedirko V, Beitler JJ, Zhou C, Gu J, et al. Gut Microbiome Associated With the Psychoneurological Symptom Cluster in Patients With Head and Neck Cancers. Cancers (Basel) (2020) 12(9):1-18. doi: 10.3390/ cancers 12092531

22. Maniakas A, Asmar M-H, Renteria Flores AE, Nayan S, Alromaih S, Mfuna Endam L, et al. Staphylococcus Aureus on Sinus Culture Is Associated With Recurrence of Chronic Rhinosinusitis After Endoscopic Sinus Surgery. Front Cell Infect Microbiol (2018) 8:150. doi: 10.3389/fcimb.2018.00150

23. Renteria AE, Maniakas A, Mfuna LE, Asmar MH, Gonzalez E, Desrosiers M. Low-Dose and Long-Term Azithromycin Significantly Decreases Staphylococcus Aureus in the Microbiome of Refractory CRS Patients. Int Forum Allergy Rhinol (2021) 11(2):93-105. doi: 10.1002/alr.22653

24. Al-Qadami G, Van Sebille Y, Le H, Bowen J. Gut Microbiota: Implications for Radiotherapy Response and Radiotherapy-Induced Mucositis. Expert Rev Gastroenterol Hepatol (2019) 13(5):485-96. doi: 10.1080/17474124.2019.1595586

25. Xu Y, Teng F, Huang S, Lin Z, Yuan X, Zeng X, et al. Changes of Saliva Microbiota in Nasopharyngeal Carcinoma Patients Under Chemoradiation Therapy. Arch Oral Biol (2014) 59(2):176-86. doi: 10.1016/j.archoralbio. 2013.10.011

26. Zhang J, Liu H, Liang X, Zhang M, Wang R, Peng G, et al. Investigation of Salivary Function and Oral Microbiota of Radiation Caries-Free People With Nasopharyngeal Carcinoma. PLoS One (2015) 10(4):e0123137. doi: 10.1371/ journal.pone. 0123137

27. Mougeot J-LC, Stevens CB, Almon KG, Paster BJ, Lalla RV, Brennan MT, et al. Caries-Associated Oral Microbiome in Head and Neck Cancer Radiation Patients: A Longitudinal Study. J Oral Microbiol (2019) 11(1):1586421. doi: 10.1080/20002297.2019.1586421

28. Reyes-Gibby CC, Wang J, Zhang L, Peterson CB, Do K-A, Jenq RR, et al. Oral Microbiome and Onset of Oral Mucositis in Patients With Squamous Cell Carcinoma of the Head and Neck. Cancer (2020) 126(23):5124-36. doi: $10.1002 /$ cncr.33161

29. Vesty A, Gear K, Biswas K, Mackenzie BW, Taylor MW, Douglas RG. Oral Microbial Influences on Oral Mucositis During Radiotherapy Treatment of Head and Neck Cancer. Support Care Cancer (2020) 28(6):2683-91. doi: 10.1007/s00520-019-05084-6

30. Hu Y-J, Wang Q, Jiang Y-T, Ma R, Xia W-W, Tang Z-S, et al. Characterization of Oral Bacterial Diversity of Irradiated Patients by High-Throughput Sequencing. Int J Oral Sci (2013) 5(1):21-5. doi: 10.1038/ijos.2013.15

31. Gao L, Hu Y, Wang Y, Jiang W, He Z, Zhu C, et al. Exploring the Variation of Oral Microbiota in Supragingival Plaque During and After Head-and-Neck Radiotherapy Using Pyrosequencing. Arch Oral Biol (2015) 60(9):1222-30. doi: 10.1016/j.archoralbio.2015.05.006

32. Hou J, Zheng H, Li P, Liu H, Zhou H, Yang X. Distinct Shifts in the Oral Microbiota Are Associated With the Progression and Aggravation of Mucositis During Radiotherapy. Radiother Oncol (2018) 129(1):44-51. doi: 10.1016/j.radonc.2018.04.023

33. Oliva M, Schneeberger PHH, Rey V, Cho M, Taylor R, Hansen AR, et al. Transitions in Oral and Gut Microbiome of HPV+ Oropharyngeal Squamous Cell Carcinoma Following Definitive Chemoradiotherapy (ROMA LA-
OPSCC Study). Br J Cancer (2021) 124(9):1543-51. doi: 10.1038/s41416020-01253-1

34. Zhu X-X, Yang X-J, Chao Y-L, Zheng H-M, Sheng H-F, Liu H-Y, et al. The Potential Effect of Oral Microbiota in the Prediction of Mucositis During Radiotherapy for Nasopharyngeal Carcinoma. EBioMedicine (2017) 18 (101647039):23-31. doi: 10.1016/j.ebiom.2017.02.002

35. Lim Y, Tang KD, Karpe AV, Beale DJ, Totsika M, Kenny L, et al. Chemoradiation Therapy Changes Oral Microbiome and Metabolomic Profiles in Patients With Oral Cavity Cancer and Oropharyngeal Cancer. Head Neck (2021) 43(5):1521-34. doi: 10.1002/hed.26619

36. Stoddard TJ, Varadarajan VV, Dziegielewski PT, Boyce BJ, Justice JM. Detection of Microbiota in Post Radiation Sinusitis. Ann Otol Rhinol Laryngol (2019) 128(12):1116-21. doi: 10.1177/0003489419862583

37. Hu Y-J, Wang Q, Jiang Y-T, Ma R, Xia W-W, Tang Z-S, et al. Exploring the Dynamic Core Microbiome of Plaque Microbiota During Head-and-Neck Radiotherapy Using Pyrosequencing. PLoS One (2013) 8(2):e56343. doi: 10.1371/journal.pone.0056343

38. Hiergeist A, Gläsner J, Reischl U, Gessner A. Analyses of Intestinal Microbiota: Culture Versus Sequencing. ILAR J (2015) 56(2):228-40. doi: 10.1093/ilar/ilv017

39. Forbes JD, Knox NC, Ronholm J, Pagotto F, Reimer A. Metagenomics: The Next Culture-Independent Game Changer. Front Microbiol (2017) 8:1069. doi: 10.3389/fmicb.2017.01069

40. Anjali K, Arun AB, Bastian TS, Parthiban R, Selvamani M, Adarsh H. Oral Microbial Profile in Oral Cancer Patients Before and After Radiation Therapy in a Cancer Care Center - A Prospective Study. J Oral Maxillofac Pathol (2020) 24(1):117-24. doi: 10.4103/jomfp.JOMFP_213_19

41. Abu Shara KA, Ghareeb MA, Zaher S, Mobacher A, Khalifa MC, Saleh SZ. Radiotherapeutic Effect on Oropharyngeal Flora in Patients With Head and Neck Cancer. J Laryngol Otol (1993) 107(3):222-7. doi: 10.1017/ S0022215100122686

42. Almstahl A, Wikstrom M, Fagerberg-Mohlin B. Microflora in Oral Ecosystems in Subjects With Radiation-Induced Hyposalivation. Oral Dis (2008) 14(6):541-9. doi: 10.1111/j.1601-0825.2007.01416.x

43. Almstahl A, Wikstrom M, Fagerberg-Mohlin B. Microflora in Oral Ecosystems and Salivary Secretion Rates-A 3-Year Follow-Up After Radiation Therapy to the Head and Neck Region. Arch Oral Biol (2015) 60 (9):1187-95. doi: 10.1016/j.archoralbio.2015.04.004

44. Arrifin A, Heidari E, Burke M, Fenlon MR, Banerjee A. The Effect of Radiotherapy for Treatment of Head and Neck Cancer on Oral Flora and Saliva. Oral Health Prev Dent (2018) 16(5):425-9. doi: 10.3290/j.ohpd.a41364

45. Keene HJ, Fleming TJ. Prevalence of Caries-Associated Microflora After Radiotherapy in Patients With Cancer of the Head and Neck. Oral Surg Oral Med Oral Pathol (1987) 64(4):421-6. doi: 10.1016/0030-4220(87)90146-0

46. Vanhoecke BWA, De Ryck TRG, De boel K, Wiles S, Boterberg T, Van de Wiele $\mathrm{T}$, et al. Low-Dose Irradiation Affects the Functional Behavior of Oral Microbiota in the Context of Mucositis. Exp Biol Med (Maywood) (2016) 241 (1):60-70. doi: 10.1177/1535370215595467

47. Stokman MA, Spijkervet FKL, Burlage FR, Dijkstra PU, Manson WL, de Vries EGE, et al. Oral Mucositis and Selective Elimination of Oral Flora in Head and Neck Cancer Patients Receiving Radiotherapy: A Double-Blind Randomised Clinical Trial. Br J Cancer (2003) 88(7):1012-6. doi: 10.1038/sj.bjc.6600824

48. Brown LR, Dreizen S, Handler S, Johnston DA. Effect of Radiation-Induced Xerostomia on Human Oral Microflora. J Dent Res (1975) 54(4):740-50. doi: $10.1177 / 00220345750540040801$

49. Chen C, Zhang Q, Yu W, Chang B, Le AD. Oral Mucositis: An Update on Innate Immunity and New Interventional Targets. J Dent Res (2020) 99 (10):1122-30. doi: 10.1177/0022034520925421

50. Villa A, Sonis ST. Mucositis: Pathobiology and Management. Curr Opin Oncol (2015) 27(3):159-64. doi: 10.1097/CCO.0000000000000180

51. Shih A, Miaskowski C, Dodd MJ, Stotts NA, MacPhail L. A Research Review of the Current Treatments for Radiation-Induced Oral Mucositis in Patients With Head and Neck Cancer. Oncol Nurs Forum (2002) 29(7):1063-80. doi: 10.1188/02.ONF.1063-1080

52. Sonis ST. Mucositis: The Impact, Biology and Therapeutic Opportunities of Oral Mucositis. Oral Oncol (2009) 45(12):1015-20. doi: 10.1016/ j.oraloncology.2009.08.006

53. Sonis ST. The Pathobiology of Mucositis. Nat Rev Cancer (2004) 4(4):277-84. doi: $10.1038 / \mathrm{nrc} 1318$ 
54. Vasconcelos RM, Sanfilippo N, Paster BJ, Kerr AR, Li Y, Ramalho L, et al. Host-Microbiome Cross-Talk in Oral Mucositis. J Dent Res (2016) 95(7):72533. doi: $10.1177 / 0022034516641890$

55. Idris A, Hasnain SZ, Huat LZ, Koh D. Human Diseases, Immunity and the Oral Microbiota-Insights Gained From Metagenomic Studies. Oral Sci Int (2017) 14(2):27-32. doi: 10.1016/S1348-8643(16)30024-6

56. Marsh PD. Dental Plaque as a Biofilm and a Microbial Community Implications for Health and Disease. BMC Oral Health (2006) 6(1):S14. doi: 10.1186/1472-6831-6-S1-S14

57. Belstrøm D. The Salivary Microbiota in Health and Disease. J Oral Microbiol (2020) 12(1):1-7. doi: 10.1080/20002297.2020.1723975

58. Peterson SN, Snesrud E, Liu J, Ong AC, Kilian M, Schork NJ, et al. The Dental Plaque Microbiome in Health and Disease. PLoS One (2013) 8(3): e58487. doi: 10.1371/journal.pone.0058487

59. Nogues JC, Pérez-Losada M, Preciado D. Review of Otitis Media Microbiome Studies: What do They Tell Us? Laryngoscope Invest Otolaryngol (2020) 5 (5):936-40. doi: 10.1002/lio2.460

60. Dimitri-Pinheiro S, Soares R, Barata P. The Microbiome of the Nose-Friend or Foe? Allergy Rhinol (Providence) (2020) 11:1-10. doi: 10.1177/ 2152656720911605

61. Man WH, de Steenhuijsen Piters WAA, Bogaert D. The Microbiota of the Respiratory Tract: Gatekeeper to Respiratory Health. Nat Rev Microbiol (2017) 15(5):259-70. doi: 10.1038/nrmicro.2017.14

62. Kumpitsch C, Koskinen K, Schöpf V, Moissl-Eichinger C. The Microbiome of the Upper Respiratory Tract in Health and Disease. BMC Biol (2019) 17(1):87. doi: 10.1186/s12915-019-0703-z

63. Kumagai T, Rahman F, Smith AM. The Microbiome and Radiation InducedBowel Injury: Evidence for Potential Mechanistic Role in Disease Pathogenesis. Nutrients (2018) 10(10):1-16. doi: 10.3390/nu10101405

64. Ma W, Mao Q, Xia W, Dong G, Yu C, Jiang F. Gut Microbiota Shapes the Efficiency of Cancer Therapy. Front Microbiol (2019) 10:1050. doi: 10.3389/ fmicb.2019.01050

65. Jiang C, Wang H, Xia C, Dong Q, Chen E, Qiu Y, et al. A Randomized, Double-Blind, Placebo-Controlled Trial of Probiotics to Reduce the Severity of Oral Mucositis Induced by Chemoradiotherapy for Patients With Nasopharyngeal Carcinoma. Cancer (2019) 125(7):1081-90. doi: 10.1002/ cncr.31907

66. Manaka A, Tokue Y, Murakami M. Comparison of 16S Ribosomal RNA Gene Sequence Analysis and Conventional Culture in the Environmental Survey of a Hospital. J Pharm Health Care Sci (2017) 3:1-16. doi: 10.1186/ s40780-017-0074-y

67. Goodwin S, McPherson JD, McCombie WR. Coming of Age: Ten Years of Next-Generation Sequencing Technologies. Nat Rev Genet (2016) 17(6):33351. doi: 10.1038/nrg.2016.49

68. Zhang X, Li L, Butcher J, Stintzi A, Figeys D. Advancing Functional and Translational Microbiome Research Using Meta-Omics Approaches. Microbiome (2019) 7(1):154. doi: 10.1186/s40168-019-0767-6

69. Albanese D, Donati C. Strain Profiling and Epidemiology of Bacterial Species From Metagenomic Sequencing. Nat Commun (2017) 8(1):2260. doi: 10.1038/ s41467-017-02209-5

70. Samra B, Tam E, Baseri B, Shapira I. Checkpoint Inhibitors in Head and Neck Cancer: Current Knowledge and Perspectives. J Investig Med (2018) 66 (7):1023-30. doi: 10.1136/jim-2018-000743

71. Ooi ML, Drilling AJ, Morales S, Fong S, Moraitis S, Macias-Valle L, et al. Safety and Tolerability of Bacteriophage Therapy for Chronic Rhinosinusitis
Due to Staphylococcus Aureus. JAMA Otolaryngol Head Neck Surg (2019) 145 (8):723. doi: 10.1001/jamaoto.2019.1191

72. Irfan M, Delgado RZR, Frias-Lopez J. The Oral Microbiome and Cancer. Front Immunol (2020) 11:591088(101560960). doi: 10.3389/fimmu.2020.591088

73. Baruch EN, Youngster I, Ben-Betzalel G, Ortenberg R, Lahat A, Katz L, et al. Fecal Microbiota Transplant Promotes Response in ImmunotherapyRefractory Melanoma Patients. Science (2021) 371(6529):602-9. doi: 10.1126/science.abb5920

74. Demmitt BA, Corley RP, Huibregtse BM, Keller MC, Hewitt JK, McQueen $\mathrm{MB}$, et al. Genetic Influences on the Human Oral Microbiome. BMC Genomics (2017) 18(1):659. doi: 10.1186/s12864-017-4008-8

75. Richards VP, Alvarez AJ, Luce AR, Bedenbaugh M, Mitchell ML, Burne RA, et al. Microbiomes of Site-Specific Dental Plaques From Children With Different Caries Status. Infect Immun (2017) 85(8):e00106-17. doi: 10.1128/ IAI.00106-17

76. Hsin CH, Tseng HC, Lin HP, Chen TH. Post-Irradiation Otitis Media, Rhinosinusitis, and Their Interrelationship in Nasopharyngeal Carcinoma Patients Treated by IMRT. Eur Arch Otorhinolaryngol (2016) 273(2):471-7. doi: 10.1007/s00405-015-3518-8

77. Kuhar HN, Tajudeen BA, Heilingoetter A, Mahdavinia M, Gattuso P, Ghai R, et al. Distinct Histopathologic Features of Radiation-Induced Chronic Sinusitis. Int Forum Allergy Rhinol (2017) 7(10):990-8. doi: 10.1002/alr.21989

78. Kamel R, Al-Badawy S, Khairy A, Kandil T, Sabry A. Nasal and Paranasal Sinus Changes After Radiotherapy for Nasopharyngeal Carcinoma. Acta Otolaryngol (2004) 124(4):532-5. doi: 10.1080/00016480410018106

79. Lu Y-T, Lu Y-C, Cheng H-C, Hsin C-H, Yang S-F, Wang P-H, et al. Chronic Rhinosinusitis After Radiotherapy in Patients With Head and Neck Cancer: A Population-Based Cohort Study in Taiwan. Int Forum Allergy Rhinol (2020) 10(5):692-7. doi: 10.1002/alr.22526

80. Rai AK, Panda M, Das AK, Rahman T, Das R, Das K, et al. Dysbiosis of Salivary Microbiome and Cytokines Influence Oral Squamous Cell Carcinoma Through Inflammation. Arch Microbiol (2021) 203(1):137-52. doi: 10.1007/ s00203-020-02011-w

Conflict of Interest: MD has received research funding from GSK, AstraZeneca, Sanofi/Regeneron, is part of the speaker's bureau for AstraZeneca and MEDA, and is founder, owner, and president of Probionase Therapies.

The remaining authors declare that the research was conducted in the absence of any commercial or financial relationships that could be construed as a potential conflict of interest.

Publisher's Note: All claims expressed in this article are solely those of the authors and do not necessarily represent those of their affiliated organizations, or those of the publisher, the editors and the reviewers. Any product that may be evaluated in this article, or claim that may be made by its manufacturer, is not guaranteed or endorsed by the publisher.

Copyright (๑) 2021 Zagury-Orly, Khaouam, Noujaim, Desrosiers and Maniakas. This is an open-access article distributed under the terms of the Creative Commons Attribution License (CC BY). The use, distribution or reproduction in other forums is permitted, provided the original author(s) and the copyright owner(s) are credited and that the original publication in this journal is cited, in accordance with accepted academic practice. No use, distribution or reproduction is permitted which does not comply with these terms. 